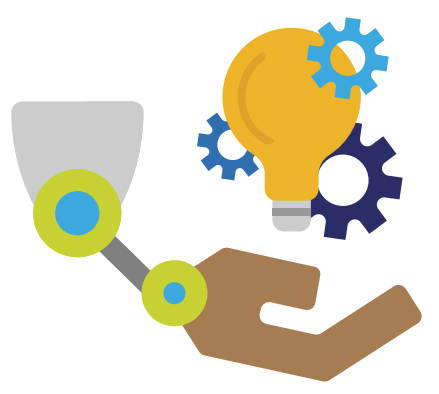

\title{
ESTUDO DE REVISÃO SOBRE GERENCIAMENTO DE ÓRTESES, PRÓTESES E MATERIAIS ESPECIAIS PARA APLICAÇÃO EM UMA INSTITUIÇÃO DE SAÚDE
}

\author{
REVIEW STUDY ON MANAGEMENT OF ORTHESIS, PROSTHESIS AND SPECIAL \\ MATERIALS FOR APPLICATION IN A HEALTH INSTITUTION
}

\begin{abstract}
Liane Lopes de Souza Pinheiro
Enfermeira da Unidade de Hemodinâmica do Hospital Universitário Onofre Lopes (HUOL). Residência em enfermagem em cardiologia e especialização em Terapia Intensiva. Atualmente é mestranda pelo Programa de Pós-graduação em Gestão e Inovação em Saúde. E-mail: lianelopesrn@hotmail.com
\end{abstract}

\section{Ana Karenina de Oliveira Paiva}

Bacharel em Ciências e Tecnologia com ênfase em Tecnologia dos Materiais (2014) pela Universidade Federal do Rio Grande do Norte (UFRN), graduada em Engenharia de Materiais (2016) e mestre pelo Mestrado Profissional em Ciências, Tecnologia e Inovação (2018) pela UFRN. E-mail: kareninapaiva@ outlook.com

\section{Jaqueline Soares da Silva}

Bacharel em Ciências e Tecnologia pela Universidade Federal do Rio Grande do Norte (UFRN), Graduanda em Engenharia Ambiental pela UFRN. E-mail: soaresjjaque@hotmail.com

\section{Angelo Roncalli de Oliveira Guerra}

Graduado em Engenharia Mecânica pela Universidade Federal do Rio Grande do Norte (1989), mestre em Engenharia Mecânica na UFRN, doutor em Engenharia Mecânica na Universidade de UMIST (University Of Manchester Institute Of Science And Technology) (1996), pós-doutor em Engenharia Virtual na University-

WVU. E-mail. profroncalli@gmail.com

\section{Custódio Leolpodino de Brito Guerra Neto}

Graduado em Odontologia (1987) pela Universidade Federal do Rio Grande do Norte (UFRN), mestre em Engenharia Mecânica (2001) e doutor em Ciência e Engenharia de Materiais (2005) pela UFRN, professor associado da UFRN no Departamento de Engenharia Biomédica. Email: custodioimplante@gmail.com

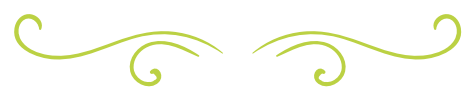




\section{RESUMO}

As Órteses, Próteses e Materiais Especiais fazem parte de uma categoria ampla de materiais utilizados na realização de procedimentos médicos, odontológicos e fisioterápicos. Atualmente, é responsável por grandes avanços na prestação da assistência à saúde. Em contrapartida, proporciona impacto financeiro, onerando a saúde, visto serem considerados produtos de alto custo. Dessa forma, existe a necessidade de desenvolver estratégias com o objetivo de estudar e descrever a importância e as atuais características do gerenciamento de Órteses, Próteses e Materiais Especiais em instituições de saúde, buscando avaliar o estado atual do conhecimento e identificar benefícios, dificuldades e desafios. A revisão da literatura foi realizada a partir de bases de dados PubMed, LILACS, SCOPUS e Google Scholar, sendo incluídos artigos científicos publicados no período de 2003 a 2019. Foi verificado que as instituições hospitalares apresentam grande fragilidade gerencial, refletindo em combinação de altos custos e baixa qualidade na assistência à saúde. Com relação à gestão de Órteses, Próteses e Materiais Especiais, existem portarias, manuais e instrumentos que orientam a gestão. Contudo, é possível verificar que são diversas as dificuldades que interferem na prática. A padronização de processos, o controle do fluxo e do estoque e a capacidade de captar e processar as informações pertinentes ao processo de trabalho são requisitos fundamentais no gerenciamento desses materiais. Como tendência, este estudo observa que a utilização da tecnologia da informação, por meio dos sistemas de gestão em saúde, é o caminho a ser adotado. Essa afirmação tem fundamento no fato de que a pesquisa realizada mostrou que os hospitais com os modelos de gestão conjugados com eficientes sistemas de TI tiveram os seguintes benefícios: melhoria do gerenciamento de Órteses, Próteses e Materiais Especiais, melhorias relacionadas ao fluxo de comunicação, padronização de processos, produção de indicadores para a gestão, rastreabilidade de dispositivos, redução dos custos. Além disso, aponta mais controle no fluxo de trabalho, minimizando os riscos de roubos e fraudes no faturamento.

Palavras-chave: Administração Hospitalar, Tecnologia da Informação, Próteses e Implantes, Equipamentos e Provisões.

\section{ABSTRACT}

OPSMs are part of a broad category of materials used to perform medical, dental and physical therapy procedures. It is currently responsible for major advances in health care delivery, in return provides financial impact, burdening health, as they are considered high cost products. Thus, the need to develop strategies to study and describe the importance and current characteristics of management of OPSMs in health institutions, seeking to assess the current state of knowledge and identify benefits, difficulties and challenges. The literature review was performed from PubMed, LILACS, SCOPUS and Google Scholar databases, including scientific articles published from 2003 to 2019. It was found that hospital institutions have great management fragility, reflecting in high costs combinations and low quality in health care. Regarding OPSMs management, there are some ordinances, manuals and instruments that guide the management. However, it is possible to verify several difficulties that interfere in practice. Process standardization, flow control, inventory and the ability to capture and process pertinent information arekey requirements in OPSMsmanagement. As a trend, it was clearly observed that the use of information technology, through health management systems, is the way to be adopted. This statement is based on the fact that research has shown that hospitals with management models coupled with efficient IT systems have had the following benefits: improved management of OPSM, 
improvements related to communication flow, process standardization, production management indicators, device traceability, cost reduction. In addition, greater workflow control was observed, minimizing the risks of bill theft and fraud.

KEYWORDS: Hospital Administration, Information Technology, Prostheses and Implants, Equipment and Supplies.

\section{INTRODUÇÃO}

As modificações do perfil epidemiológico da população, que se caracterizam pelo envelhecimento das pessoas e predomínio das doenças crônicas não transmissíveis associado à implantação do Sistema Único de Saúde (SUS), começaram a exigir que a gestão dos serviços de saúde passe por mudanças de forma a garantir a eficiência e o acesso aos serviços de forma universal (PEREIRA, 2015). Esse mesmo processo gerou notórias mudanças na gestão hospitalar, tanto nos aspectos referentes à redefinição do seu papel no sistema de atenção à saúde como na sua reorganização interna de trabalho, com o surgimento de novos modelos de gestão hospitalar divulgados na literatura. Dessa forma, cada hospital possui seu próprio modelo implantado, buscando excelência para procedimentos de alta complexidade, tecnologia, alto nível de confiabilidade e qualidade, objetivando a total assistência ao paciente, tendo como desafio a garantia do equilíbrio financeiro (DEUS; MELO, 2015). Assim, atualmente, essas instituições hospitalares são consideradas organizações complexas, com profissionais especializados que desenvolvem diversas atividades em diferentes níveis de atenção.

No exercício dos trabalhadores da saúde, é utilizada uma variedade de materiais com muita tecnologia, o que tem contribuído para a elevação dos custos. O gerenciamento de tais materiais, com os recursos humanos e financeiros, é a base de sustentação do hospital, sendo o primeiro considerado o mais complexo quando comparado ao de outros segmentos. Isso se deve ao fato de as despesas com materiais representarem em torno de $25 \%$ dos orçamentos correntes (MORAES; RABIN; VIÉGAS, 2018). Entre os materiais utilizados, cada vez mais, as Órteses, Próteses e Materiais Especiais (OPMEs) assumem grande importância econômica nas contas dessas instituições (BRASIL, 2015).

As OPMEs fazem parte de uma categoria ampla de materiais, com um universo extremamente diverso de produtos utilizados na realização de procedimentos médicos, odontológicos e fisioterápicos, contribuindo para diagnóstico, tratamento, reabilitação ou monitoração de pacientes (BRASIL, 2015). Em geral, caracterizam-se pelo elevado grau de diversidade e distinção tecnológica, sendo responsáveis por grande avanço na assistência à saúde da população, no contexto mundial, estando diretamente relacionados à evolução dos procedimentos cirúrgicos (ALENCAR, 2016).

Esse fato se evidencia atualmente por meio das diferentes especialidades médicas que dispõem de OPMEs complexas e tecnologias avançadas que, associadas ao surgimento das cirurgias endovasculares e intervenções percutâneas, possibilitaram a substituição de cirurgias convencionais por tratamentos inovadores e permitiram melhor prognóstico e qualidade de vida (CAMARGO, 2017). Contudo, apesar do ganho na prestação de assistência à saúde, a utilização das diversas OPMEs proporciona impacto, onerando a saúde, visto serem considerados produtos de alto custo (ALENCAR, 2016). Diante desse contexto, o gerenciamento desses recursos materiais tem sido motivo de preocupação principalmente nas organizações de saúde do setor público, pois, devido a orçamentos restritos, necessitam de mais controle do consumo e dos custos para que não privem funcionários e pacientes do material necessário (GARCIA et al., 2012). 
Na prática, é possível perceber que o setor público tem uma tendência em direcionar os problemas de falta de materiais médico-hospitalares ou problemas de estoques à falta de recursos financeiros. Mas não é só a ausência de recursos financeiros que gera problemas no abastecimento dos materiais médico-hospitalares, a deficiência no controle de estoque também pode ter grande parcela de responsabilidade nesse processo, causando desperdícios e má utilização, gerando problemas de abastecimento (RAIMUNDO; DIAS; GUERRA, 2015). Outra dificuldade enfrentada é a não padronização dos modelos (i.e. processos). Como consequência, gera a ocorrência de retrabalho, desperdício de tempo, materiais e mão de obra, interferindo diretamente nos custos e na qualidade final do atendimento (SILVA et. al., 2018).

A gestão de tais OPMEs permeia grande parte dos processos hospitalares, desde 0 agendamento do procedimento até a contabilização das informações, passando por logística, consumo e faturamento. Esse fluxo contribui para que suas características assistenciais e de comercialização sejam consideradas complexas, pois envolve pessoas, processos, sistemas de informação e fornecedores (MORAES; RABIN; VIÉGAS, 2018). Gerenciar materiais implica planejar, executar e controlar de forma eficiente e econômica o fluxo de materiais, desde as especificações dos materiais até a sua entrega (GARCIA et al., 2012). Esse processo se torna importante, pois se evidencia que, quando falhas ocorrem em algum ponto, o custo da operação permanece ativo. Isso se dá devido aos recursos humanos, tecnológicos e logísticos que continuam instalados, sendo acrescidos os custos de ociosidade de sala de cirurgia, preparo inadequado e privação do material necessário ao paciente (GARCIA et al., 2012; MORAES; RABIN; VIÉGAS, 2018).

Esse contexto faz surgir a necessidade de desenvolver estratégias com o objetivo de aprimorar o gerenciamento do uso das
OPMEs, de forma a melhorar o controle dos gastos financeiros, aproximar todos os envolvidos e agilizar o fluxo de comunicação. Isso envolve ter mais controle no processo de solicitação, dispensação, rastreabilidade e gerar informações mais precisas sobre o uso desses dispositivos (SILVA; LIMA, 2015).

Alguns dos desenvolvimentos recentes que observamos na prestação de cuidados de saúde são os novos modelos de negócios que visam melhorar o planejamento de fluxos de trabalho em todo o hospital usando tecnologia da informação, gerenciamento de operações e avançadas técnicas de análise de dados (CAÑAMARES-ORBIS et al., 2014). Tal reorganização reflete a preocupação da realidade nacional brasileira na qual o gasto anual com OPMEs é de R $\$ 20,5$ bilhões (ITO, 2015). Esse valor possui uma tendência à continuidade do aumento dos custos nos próximos anos, pois a utilização desses insumos possui sua predominância nos setores hospitalares de maior expansão, destacando-se: unidades de centro cirúrgico, hemodinâmica, diagnósticos por imagem e terapia intensiva (MORAES; RABIN; VIÉGAS, 2018). Com isso, há previsão de impacto direto na equação de custos sobre as receitas do governo e das operadoras de saúde suplementares (FONSECA JÚNIOR, 2018).

Diante de tais elementos, o objetivo deste trabalho foi realizar uma revisão bibliográfica sobre gerenciamento de OPMEs em instituições de saúde. Este estudo busca avaliar o estado atual do conhecimento e identificar benefícios, dificuldades, desafios e tendências dos modelos de gestão hospitalar.

\section{REVISÃO BIBLIOGRÁFICA}

Nesta seção, será apresentado o referencial teórico acerca do gerenciamento de OPMEs em instituições de saúde. Para isso, os assuntos foram divididos em tópicos que tratam sobre gestão hospitalar, evolução histórica das OPMEs, gestão das OPMEs e 
sistemas de informação como instrumento de gestão em saúde.

\section{Gestão hospitalar}

A evolução da gestão hospitalar está diretamente relacionada à história dos hospitais e da medicina. Os hospitais no Brasil, como em qualquer outro país, foram administrados por religiosos, médicos, enfermeiros ou pessoas da comunidade, devido ao fato de não serem vistos como uma empresa e sim como uma "instituição de caridade". Nem sempre o gestor conhecia a prática hospitalar, nem as técnicas de gerenciamento. $\mathrm{Na}$ verdade, não existia a figura do gestor, mas, sim, a função de manter a estrutura física e de cuidar das despesas com os poucos recursos existentes (SEIXAS; MELO, 2004). Essa realidade se inseria no perfil de instituição hospitalar que, historicamente, esteve centrada na figura do médico como um instrumento de cura (NEUFELD, 2013).

Atualmente, com a modificação do perfil das organizações hospitalares, associada ao novo contexto de políticas públicas, particularmente a partir da instituição do SUS, a gestão em saúde se volta para um padrão de atenção disponibilizada à população, passando a visar à saúde universal, segura e de qualidade, que, em função do surgimento de novas tecnologias em saúde, alimenta custos crescentes no setor e exige práticas de gestão inovadoras e apropriadas (LORENZETTI; GELBCKE; VANDRESEN, 2016). Nesse novo cenário, os hospitais se caracterizam por serem organizações que possuem complexas estruturas físico-funcionais e uma diversidade de recursos humanos e materiais especializados, necessários às intervenções técnicas realizadas no âmbito do tratamento e da prevenção de danos à saúde (SILVA et al., 2019). Dessa forma, as diversas organizações de saúde têm como missão a eficácia e a qualidade dos serviços prestados à população, além da garantia da sustentabilidade financeira (LORENZETTI; GELBCKE; VANDRESEN, 2016).

Dentre as questões que colaboram com o incremento da complexidade na gestão hospitalar, destaca-se a ampliação da base de clientes potenciais, com o aumento da população idosa e a prevalência de doenças crônicas. Isso implica aumento na demanda dos hospitais, independentemente do tipo de gestão, pública ou privada, agravando um quadro de escassez de recursos médicos e de longas esperas por atendimento. Somado a isso, podemos destacar outros fatores capazes de acentuar o desafio da gestão hospitalar, como, por exemplo, a ausência ou a ineficiência de mecanismos de avaliação do desempenho da gestão, as divergências e os conflitos interpessoais existentes entre os profissionais de saúde, a deficiência na comunicação e a definição pouco precisa dos processos de trabalho. Todos esses fatores dificultam a prestação de serviços eficientes e de qualidade (FARIAS; ARAÚJO, 2017).

Diante de toda essa problemática envolvendo a gestão hospitalar, a temática tem assumido um papel cada vez maior na agenda dos gestores, profissionais de saúde e usuários. Esse fato é evidenciado pelas crescentes iniciativas de adoção de diretrizes e protocolos clínicos, de acreditação hospitalar, de definição de padrões, de busca de mais segurança para os pacientes e de qualificação da assistência hospitalar (AZEVEDO et al., 2017). Além dessa perspectiva, no setor público, têm sido possíveis a criação e a implementação de novos processos e produtos, bem como de novos métodos e técnicas de prestação de serviços, o que perpassa pelo sentido da inovação nessa área (CAÑAMARES-ORBIS et al., 2014).

Com isso, hoje se discute a inovação como uma ferramenta gerencial básica, também considerada como um instrumento de transformação de culturas organizacionais e reconhecida como o ingrediente mais importante em qualquer economia moderna (VILELA PINTO; NOSSA; TEIXEIRA, 2015). 
Em sua definição geral, a inovação é um produto/processo novo ou melhorado que difere significativamente dos produtos ou processos anteriores da unidade (OECD/ EUROSTAT, 2018). No contexto específico dos serviços públicos, a inovação é definida como a implementação de uma mudança significativa no modo de uma organização operar ou nos produtos que ela fornece. Inovações compreendem mudanças novas ou significativas em serviços e bens, processos operacionais, métodos organizacionais, ou na forma que uma organização se comunica com seus usuários. A inovação, tanto tecnológica quanto organizacional, tem trazido incontáveis benefícios para o Sistema de Saúde, uma vez que tem sido vista como a solução para que ele se torne capaz de responder aos novos desafios que enfrenta (VIEIRA, 2016).

Nessa perspectiva de mudança e aprimoramento da gestão hospitalar, são evidenciadas na literatura práticas orientadas a satisfazer as necessidades de solucionar ou contornar problemas interpessoais e de definição de processos. Exemplos disso são os colegiados gestores (gestão participativa), os modelos de acreditação hospitalar, as metodologias de gerenciamento de projetos e o investimento em inovação tecnológica. A gestão participativa traduz a busca da gestão hospitalar por práticas mais dialógicas e democráticas, numa forma de administração horizontal, em que se cria um espaço, preferencialmente físico, destinado à realização de reuniões orientadas ao debate de ideias e à identificação e à resolução de problemas.

A premissa dos colegiados gestores é o aprimoramento da comunicação institucional, o que pode repercutir positivamente em demais fatores, tais como o faturamento. Enquanto a gestão participativa focaliza o aspecto da comunicação, a acreditação hospitalar privilegia o aprimoramento dos processos hospitalares. A partir de um diagnóstico dos processos de trabalho da instituição, são implementadas medidas voltadas ao aprimoramento dos referidos processos (FARIAS; ARAÚJO, 2017). A utilização de gestão de projetos na gestão hospitalar apresenta como uma de suas vantagens a diversidade de métodos que tem a oferecer ao específico contexto hospitalar. Nessa perspectiva, diversas instituições de saúde estão adotando cada vez mais o método Lean Six Sigma (LSS), dedicado ao aumento da agilidade e da eficiência nos negócios (GIJO; ANTONY, 2014).

Outro tema que emerge como estratégia para a gestão hospitalar é o aspecto da inovação tecnológica. Esta surge para auxiliar no aprimoramento do processo gerencial, uma vez que apresenta a informação e a comunicação, em tempo real, como as principais ferramentas integradoras dos processos produtivos, impactando positivamente no atendimento ao paciente e à gestão do cuidado (AGUIAR; MENDES, 2016).

\section{Evolução histórica das OPMEs}

São muito antigos os registros, particularmente, do uso de próteses e órteses pelos seres humanos. Os primeiros registros datam de 2750 a 2625 a.C. quando homens utilizando próteses e órteses foram retratados em pinturas da quinta dinastia egípcia e o dedo de um pé, feito de madeira, foi encontrado durante o Império Novo (EDELSTEIN; BRUCKNER, 2006).

Apesar desses registros, sabe-se que foram as guerras que impulsionaram a produção de órteses e próteses para que os soldados pudessem ser reabilitados e, em alguns casos, voltassem para o campo de batalha. Um registro feito por Plínio, romano cientista e historiador que viveu entre 23 a 79 a.C., descreve a história do general romano Marcus Sergio (218 a 210 a.C.), que teve sua mão direita cortada durante uma batalha, sendo esta substituída por uma de ferro para que pudesse segurar o escudo e retornar ao combate. Nessa época, as próteses 
eram utilizadas apenas para substituir os membros (NORTON, 2007).

$\mathrm{Na}$ atualidade, além de substituírem membros amputados, elas melhoram a funcionalidade, passando também a dispor de tecnologias que permitem ser implantadas cirurgicamente em diversos outros sistemas do corpo humano, promovendo sobrevida e qualidade de vida. Um exemplo são os implantes cocleares, stents vasculares, estimuladores cerebrais, desfibriladores, próteses ortopédicas cirurgicamente implantadas, implantes dentários, bem como outros dispositivos (BRASIL, 2016).

Dessa evolução, surgiu a sigla OPMEs para caracterizar os insumos utilizados na assistência à saúde e relacionados a uma intervenção médica, odontológica ou de reabilitação diagnóstica ou terapêutica. Tais insumos são considerados complexos, levando-se em conta principalmente a diversidade dos produtos existentes e o uso de tecnologias altamente sofisticadas (BRASIL, 2016).

De forma mais detalhada, as órteses são peças ou aparelhos de correção ou complementação de membros ou órgãos do corpo. Também são definidas como qualquer material permanente ou transitório que auxilie as funções de um membro, órgão ou tecido. A prótese é considerada peça ou aparelho de substituição dos membros ou órgãos do corpo. Compreende qualquer material permanente ou transitório que substitua total ou parcialmente um membro, órgão ou tecido. Os materiais especiais são quaisquer materiais ou dispositivos de uso individual que auxiliam em procedimento diagnóstico ou terapêutico e que não se enquadram nas especificações de órteses ou próteses (BRASIL, 2016).

Atualmente, a indústria desses dispositivos é a que mais cresce devido à inovação e ao rápido avanço de suas tecnologias. Sabe-se que cerca de 6\% das pessoas nos países industrializados e $10 \%$ da população da América experimentaram uma OPME para reconstruir as funções do corpo, alcançar melhor qualidade de vida ou expandir a longevidade. A ampla gama desses dispositivos médicos desempenha um papel importante em vários aspectos dos serviços de saúde, como diagnóstico, tratamento, prevenção e reabilitação (ALSHAGATHRH et al., 2018). Em geral, esses dispositivos se caracterizam pelo elevado grau de diversidade e distinção tecnológica, um ciclo de vida curto no qual, mesmo produtos lançados recentemente, podem se tornar obsoletos ou concorrencialmente inviáveis antes que sejam conhecidos seus potenciais benefícios ou malefícios (BRASIL, 2015).

No Brasil, tais dispositivos têm sido utilizados de forma crescente nos setores público e privado de saúde. Isso porque foi ampliada a demanda por essas tecnologias, tanto no diagnóstico como no tratamento, em face das mudanças demográficas e epidemiológicas (em especial, o aumento do número de idosos) e sociais (traumas por violência urbana e acidentes de trânsito) (BRASIL, 2015). Com relação ao setor produtivo de dispositivos, o mercado de produtos de empresas brasileiras ainda é discreto frente aos principais países produtores de dispositivos médicos do mercado mundial (BRASIL, 2015). Essa realidade atual tem sido responsável por significativos aumentos nos custos relacionados à assistência à saúde (BRASIL, 2015), o que tem tornado as OPMEs itens hospitalares que geram bastante preocupação no que diz respeito ao seu gerenciamento (NARDINO; DALCUL; GIL, 2011).

\section{Gestão de OPMEs}

O surgimento da gestão de materiais decorreu da necessidade de planejar, executar e controlar de forma eficiente e econômica o fluxo de materiais, desde as especificações dos materiais até a sua entrega (GARCIA et al., 2012). No processo de gestão de OPME, diversos pontos podem ser considerados críticos para o 
atendimento adequado ao paciente, tais como: a definição pelo médico do melhor material indicado para o tratamento proposto; a operacionalização da informação transmitida pelo médico, a organização da logística de compra e a autorização de uso desses materiais pelas áreas administrativas, o recebimento, o preparo e a disponibilização do material para o uso que é de responsabilidade da área assistencial (MORAES, 2014).

Nesse processo, supera-se o desafio de gerir eficientemente as OPMEs quando se alcançam os objetivos de garantir: segurança do paciente, eficiência operacional, redução de desperdício e variabilidade, relações comerciais e técnicas harmoniosas, oferta de uma boa relação custo-benefício para os produtos, eliminação do risco de glosas/ atrasos no faturamento, alcance de confiança e resolubilidade (BRASIL, 2016). Para que seja possivel contemplar todos esses objetivos, faz-se necessária uma equipe multiprofissional, com um desempenho afinado de suas atividades, com processos padronizados e um conhecimento técnico-científico muito específico (MORAES, 2014). Isso porque a gestão de OPMEs permeia grande parte dos processos hospitalares, desde o agendamento do procedimento até a contabilização das informações, passando pela logística, pelo consumo e pelo faturamento, o que envolve pessoas, processos, sistemas de informação e fornecedores, tornando essa gestão complexa e desafiadora (MORAES; RABIN; VIÉGAS, 2018).

Diante da complexidade na gestão e dos custos elevados das OPMEs, atualmente, existem legislações específicas, normas e manuais, que determinam diversos critérios para favorecer a boa gestão desses itens, particularmente em instituições públicas, podendo ser destaque a portaria $N^{0} 1.302$ de 01 agosto de 2017. Esse documento redefine os critérios para aquisição, recebimento, utilização, monitoramento, controle e gerenciamento de OPMEs pelos hospitais e institutos federais subordinados à
Secretaria de Atenção à Saúde do Ministério da Saúde (SAS/MS), devendo esses procedimentos observar as diretrizes do Manual de Boas Práticas em Órteses, Próteses e Materiais Especiais (OPME), do Ministério da Saúde (BRASIL, 2017).

Esse manual sugere diretrizes e propõe a uniformização das atividades de aquisição, solicitação, recebimento, armazenagem, dispensação, utilização e controle de OPMEs, visando organizar o fluxo do processo de trabalho, em consonância com os meios que assegurem a adoção de boas práticas. Destacam-se as melhores práticas para serem desenvolvidas desde o planejamento da aquisição até as auditorias internas (BRASIL, 2016). O manual também estabelece que as aquisições são precedidas da apresentação de "Requisição" e "Termo de Referência", elaborados por servidor com qualificação profissional, devendo o referido termo ser avaliado e aprovado por comissão especial nomeada pelo diretor da unidade hospitalar. Os instrumentos convocatórios de licitação e os contratos deles decorrentes devem observar as disposições legais estabelecidas para esse fim e normas infralegais que regem as contratações públicas. As licitações são realizadas na forma eletrônica, regulamentada.

Toda OPME destinada à unidade hospitalar é entregue nos respectivos almoxarifados centrais ou depósitos. $\bigcirc$ recebimento, a armazenagem e a distribuição desses produtos, no âmbito de cada unidade, são de responsabilidade dos almoxarifes, que devem, após o recebimento dos materiais, realizar o registro das informações no Sistema Integrado de Administração Financeira do Governo Federal (SIAFI) e no sistema informatizado específico de cada hospital.

A distribuição de OPMEs ocorre após a solicitação do profissional de saúde responsável pelo procedimento cirúrgico ao almoxarifado central, com antecedência mínima de 48 horas, em caso de procedimentos eletivos. $\bigcirc$ controle na utilização de OPMEs é de responsabilidade exclusiva dos 
profissionais de saúde envolvidos na realização do procedimento, que devem registrar no relato cirúrgico, na folha de consumo e no prontuário do paciente todas as informações sobre as OPMEs utilizadas, ou que não foram utilizadas, discriminando os motivos (BRASIL, 2016).

No que diz respeito ao faturamento em instituições conveniadas ao SUS, O Sistema de Gerenciamento da Tabela de Procedimentos, Medicamentos e OPM do SUS (SIGTAP/SUS) é um instrumento indispensável para a gestão de OPME. Esse sistema de gerenciamento se destaca por contemplar procedimentos médicos, medicamentos, órteses, próteses e materiais especiais oferecidos pelo SUS e permitir compatibilizar os códigos dos procedimentos com as OPMEs autorizados para cada um deles, além de fornecer os valores pagos pelos procedimentos e seus respectivos dispositivos médicos (CAMARGO, 2017). Dessa forma, os valores de todos os procedimentos no SUS são fixos e as descrições das órteses e próteses são genéricas, sem especificação de marca ou tipo (BRASIL, 2015).

Apesar desses diversos documentos portarias, manuais e instrumentos - que orientam a gestão de OPMEs, ainda é possível verificar que são inúmeras as dificuldades que interferem na prática. Sabe-se que não é recente o conhecimento que as dificuldades financeiras relacionadas ao uso de OPMEs pelas instituições hospitalares ultrapassa os altos custos desses itens. O problema vai além, envolve também: gerenciamento ineficiente decorrente da falta de informação de qualidade; conflitos de interesse entre os atores; falta de controle de estoque; falta de padronização dos processos; desperdícios e má utilização desses materiais; baixa qualificação dos profissionais; e o ineficiente planejamento logístico nas organizações de saúde, sobretudo as públicas (NARDINO; DALCUL; GIL, 2011; FERREIRA JUNIOR, 2013).

A consequência disso são as constantes rupturas no abastecimento de suprimentos, acarretando prejuízos drásticos na assistência à saúde, como a precariedade do atendimento de urgência, a ausência de materiais e medicamentos para atender as demandas, ou a insuficiência de recursos para suprir as necessidades da sociedade com o sistema de saúde (SILVA, 2015). Portanto, é possível identificar no setor hospitalar falhas no planejamento efetivo, além de ineficiências na gestão de suprimentos, que acarretam perda financeira, atrasos de dispensação de materiais e insumos, falta ou excesso de estoques (SILVA et al., 2018).

Um dos problemas que tomou destaque nos últimos anos foram os conflitos de interesses envolvendo a prescrição de OPMEs. Um exemplo disso é a permissão, por parte de alguns médicos, do assédio financeiro de fabricantes, fornecedores e distribuidoras de materiais, que oferecem ganhos econômicos em troca da utilização de marcas comerciais específicas, independentemente da efetiva indicação técnica e da necessidade do paciente. Isso contribui para o uso abusivo de OPMEs e pode influenciar negativamente na adesão desses profissionais a protocolos clínicos e na utilização de outros recursos existentes nos hospitais, o que dificulta a implementação de processos padronizados nas instituições de saúde (SILVA et al., 2018).

Um fator que dificulta a gestão é a existência de assimetrias de informações relacionadas às OPMEs de ordem técnica e econômica. Desse modo, de um lado, está a não de uma padronização de denominações ou nomenclaturas, o que inviabiliza a identificação de produtos e, de outro, a ausência de bancos de preços confiáveis (BRASIL, 2015). A falta de padronização dificulta a procura e a escolha adequada do produto por parte da instituição, complicando a comparação de preços durante a sua compra (SILVA et al., 2018). Além disso, na realidade brasileira, a compra de materiais em grande escala e negociação de preços com fornecedores é atravancada pelo fato de que os hospitais, em sua grande maioria, têm estruturas 
pequenas e fragmentadas, o que acarreta na compra de produtos para a saúde com preços elevados (CAMARGO, 2017).

Somado a isso, dentre as principais fragilidades da gestão na saúde, particularmente no setor público, destaca-se o despreparo dos profissionais para o exercício da administração. Como consequência, a maioria das organizações públicas de saúde, tal como a maioria das organizações no geral, tem problemas gerenciais (REIS, 2017). Como exemplo, podemos citar o descontrole do estoque que é identificada pela falta e/ou pelo excesso de material, responsável pelo aumento desnecessário de despesa e/ ou a escassez dos insumos, que gera perda de lucro e, mais grave, a ruptura no atendimento ao cliente (SILVA; LIMA, 2015).

O principal motivo da ausência de controle nos estoques hospitalares é não haver informações de qualidade e em quantidade suficiente. Nesse contexto, podemos citar as falhas mais comuns, a saber: previsão de consumo, prazos de entrega, alternativas de distribuições em estoques existentes nos almoxarifados e depósitos. Atualmente, sabe-se que quanto mais precisas forem as informações disponíveis, maiores serão as possibilidades de determinar o volume adequado de estoques para atender as atividades da organização. Com isso, o conhecimento dos custos é de fundamental importância e, sem um sistema de informação que facilite o processo, é praticamente impossível gerenciá-los (SCHROEDER; SCHIOCHET, 2018).

Atrelado a isso, outra dificuldade apresentada é a impermeabilidade das instituições hospitalares às mudanças, sobretudo de cunho gerencial. Isso ocorre devido à fragmentação da prática clínica, com baixo grau de interação entre as especialidades e profissões, subordinação dos usuários aos serviços e pouca capacidade de governo dos gestores para atuar sobre as corporações, especialmente em relação aos médicos. Com isso, percebe-se uma lentidão na incorporação de novas tecnologias de informação e processos de gestão e de organização do trabalho (REIS, 2017). Existem barreiras de legislação no setor público que restringem a agilidade necessária, ademais, há uma alta rotatividade dos gestores das esferas federativas em função da relação com os processos partidários e eleitorais, o que gera descontinuidade, permanentes recomeços e desmotivação dos profissionais e trabalhadores (REIS, 2017).

\section{Tecnologia e Sistemas de Informação como instrumento de gestão em saúde}

Nas últimas décadas, houve um intenso processo de transformação e de inovação tecnológica sem precedentes na área da saúde. De fato, a tecnologia ultrapassou o processamento-padrão de dados para funções administrativas comuns em todas as organizações, tais como: recursos humanos, folhas de pagamento, sistemas de contabilidade, entre outros, e atualmente desempenha um papel fundamental tanto no cuidado ao paciente (ex. na interpretação do eletrocardiograma) como em escalas de trabalho, prescrição, relatório de resultados e sistemas de prevenção (PINOCHET; LOPES; SILVA, 2014).

A tecnologia da informação é definida como todo e qualquer dispositivo que possua a habilidade para tratar e processar dados ou informações de uma maneira sistêmica, estando contida no produto ou processo. Qualquer tecnologia da informação deve proporcionar ao usuário o domínio efetivo da informação, além de simplificar os passos das atividades (CRUZ, 2011). Para Pinochet, Lopes e Silva (2014), o uso das tecnologias da informação e comunicação possibilita melhorias nas rotinas de uma gestão hospitalar e redução de custos, com aumento de controle de situações decisivas tanto para a sobrevivência de pacientes quanto para a saúde econômico-financeira da própria instituição. 
Uma solução organizacional e administrativa passa pela implantação de um sistema de informação conjugado. Na área da saúde, os sistemas de informação têm um grande potencial na redução de erros, aumentando a legibilidade, cortando custos desnecessários e ampliando a qualidade dos cuidados de saúde. Um bom sistema de informação contribui positivamente para a segurança do paciente, a eficiência do fluxo de trabalho e o apoio à decisão no ponto de atendimento (KUSHNIRUK; PATEL, 2004).

Atualmente, utiliza-se o termo "sistemas de informações gerenciais" para definir os sistemas desenvolvidos que ajudam as empresas a alcançar metas e objetivos. Nesse sentido, a adoção de um Sistema de informação para a Gestão da Qualidade, com uma arquitetura de informação que favoreça a qualidade e agregue valor aos resultados, é uma opção estratégica e reflete na melhoria e na promoção da sustentabilidade dos serviços de saúde (SOUZA, 2013).

No que se refere à inovação tecnológica, a tecnologia da informação e os sistemas de informações têm funcionado como elo entre as atividades relativas ao processo assistencial e aqueles referentes ao processo administrativo, uma vez que apresentam a informação e a comunicação como as principais ferramentas integradoras dos processos produtivos (AGUIAR; MENDES, 2016). Isso tende a atender a um dos grandes problemas das instituições de saúde do SUS, que decorre do fato de setores clínicos e administrativos não se identificarem como uma cadeia produtiva. Em geral, o que os setores clínicos produzem não é transparente para os setores ligados à administração e vice-versa, gerando desconhecimento e desencontro de informações, dificultando a gestão. Dessa forma, a tecnologia da informação e os sistemas de informações são citados como ferramentas importantes para a gestão em saúde (PINOCHET; LOPES; SILVA, 2014).

\section{METODOLOGIA}

Realizou-se uma revisão bibliográfica, com o objetivo de estudar e descrever a importância e as atuais características do gerenciamento de OPMEs em instituições de saúde, buscando avaliar o estado atual do conhecimento e identificar benefícios, dificuldades e desafios. Assim, a primeira etapa desta pesquisa correspondeu à investigação com base em artigos publicados no portal de periódicos CAPES, na base de dados PubMed, LILACS, SCOPUS e Google Scholar. Procedendo com os trabalhos para se obter um maior refinamento dos filtros, decidiu-se por adotar um critério de inclusão, tal como: artigos científicos publicados no período de 2003 a 2019 sobre o assunto, os quais estão disponíveis nos idiomas português, inglês e espanhol. Foram realizadas, também, pesquisas junto ao Ministério da Saúde do Brasil no mesmo período.

Visando focar em termos mais específicos, em cada base foi utilizado o recurso "filtrar meus resultados", por tópicos. Considerando o objetivo maior desta pesquisa, foi selecionado um conjunto de termos que, no entendimento dos autores deste trabalho, são os mais adequados para englobar o maior número de artigos de interesse e relevância sobre o tema proposto. Assim, houve o cruzamento das palavras-chaves indexadas nos descritores em ciências da saúde (DeCS): administração hospitalar, próteses e implantes, gestão de recursos hospitalar, administração de materiais no hospital, sistema de informação, totalizando em torno de 1200 artigos. Também, foram utilizados os mesmos descritores na língua inglesa, de acordo com a base de dados. A busca foi realizada a partir do sítio eletrônico, em janeiro de 2019.

Realizados os cruzamentos entre os descritores, e após uma pré-seleção desses estudos por meio da leitura dos títulos e dos resumos, quando necessário, foram selecionadas 58 publicações. Os artigos foram lidos na íntegra, a fim de identificar a adequação 
destes aos critérios de inclusão destinados a esta revisão. A configuração da metodologia desta revisão está esquematizada na Figura 1.

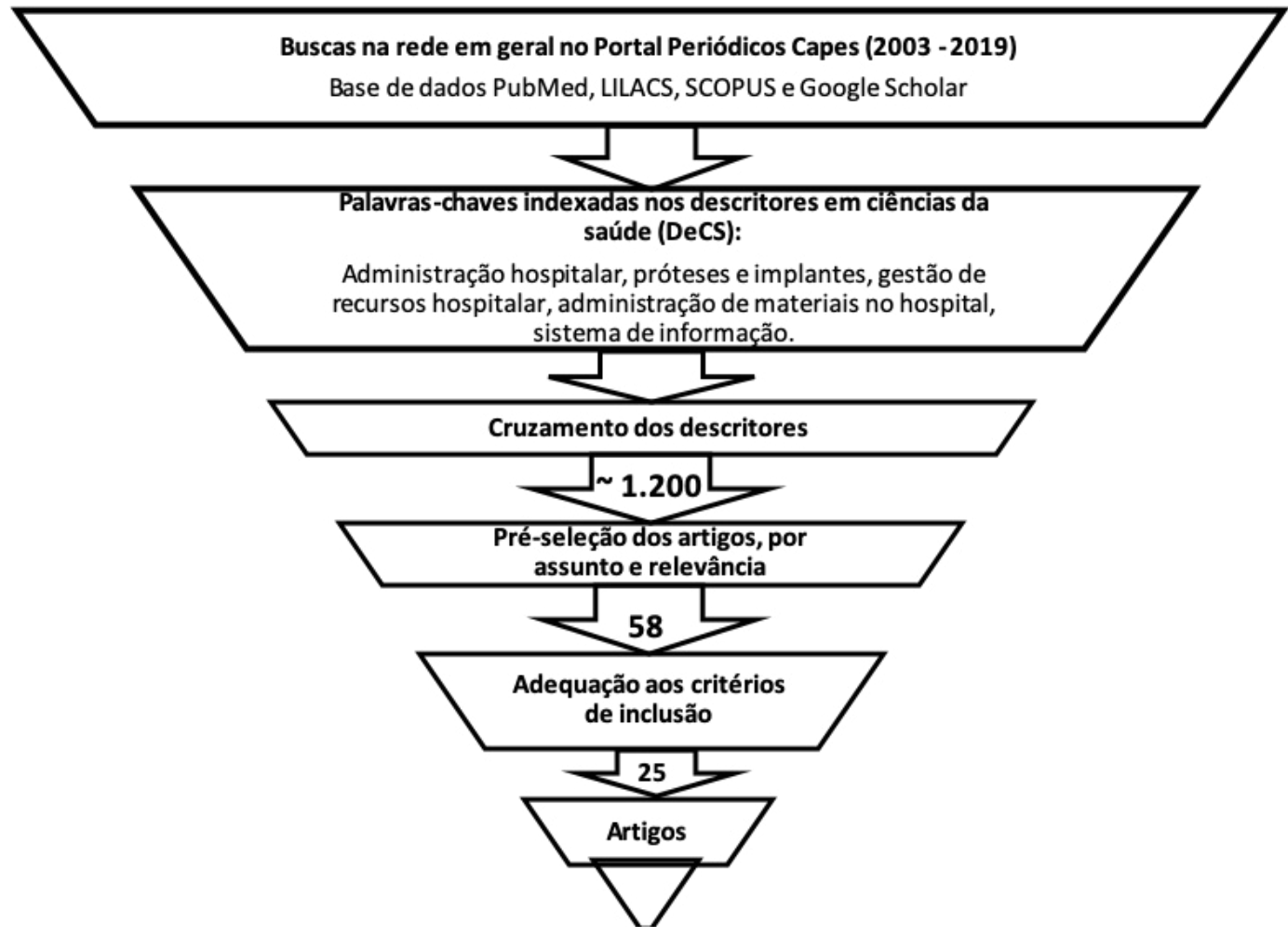

Figura 1 - Etapas da metodologia utilizada nas buscas por artigos na área específica.

Fonte: Elaborado pelos autores (2019).

Após as etapas de pré-seleção dos artigos, foram excluídos 33, chegando-se a uma amostra final de 25 artigos. Desse total, o maior número de publicações ocorreu em 2018 (5 artigos). No que se refere aos idiomas dos artigos, 5 eram em inglês, 19 em português e 1 em espanhol. Em relação às bases de dados, foram encontrados 4 artigos na PubMed, nos anos de 2016 e 2018; no Google Scholar, foram selecionados 20 artigos, publicados entre os anos 2003 de 2018; e na Scopus 1 artigo, referente ao ano de 2016.

\section{RESULTADOS E DISCUSSÃO}

Segundo Lorenzetti, Gelbcke e Vandresen (2016), a rede hospitalar brasileira tem o diagnóstico da existência de inúmeros estabelecimentos de saúde com grande fragilidade gerencial e que estão longe de atender o exigido para oferecer uma assistência resolutiva, adequada e otimizada. Como consequência disso, segundo Souza e Carvalho (2015), as organizações de saúde apresentam uma combinação de altos custos e qualidade insuficiente, gerando ansiedade e insatisfação aos clientes, médicos e demais prestadores de serviços, planos de saúde, fornecedores e governo. Esse fato evidencia as claras limitações e dificuldades relacionadas aos métodos tradicionais de gestão em saúde, confirmadas por esses autores.

Na tentativa de mudança dessa realidade, Souza e Carvalho (2015) afirmam que as instituições hospitalares vivenciam um momento singular, buscando reinventar suas práticas, otimizar custos e atender a necessidades e expectativas de clientes e fontes pagadoras, em um processo de profissionalização do setor para melhoria de resultados. Essa modificação de forma de gestão também é abordada por Alves, Guerra Neto e Hékis (2018) quando afirmam que tanto os espaços 
públicos quanto os privados passam por um processo de transformação no modelo de gestão e organização da produção de bens e serviços de saúde. Assim, há uma nova concepção sobre gestão e desempenho, mais voltada para a qualidade do produto e dos serviços oferecidos, a minimização de custos e o compromisso no atendimento de populações e clientelas específicas.

Complementando essa concepção, Azevedo et al. (2017) abordam que o debate sobre gestão em saúde no âmbito internacional e no Brasil trazem como foco a importância da gestão hospitalar nas agendas governamentais, como corolário quase natural das políticas de contenção de gastos e busca de maior eficiência para o setor. Para os autores, o principal alvo dos hospitais é criar um novo modelo de gestão, fundado em novas estruturas, regras, novos incentivos, ferramentas e práticas gerenciais que garantam serviços de saúde mais eficientes, com melhores resultados, oferecendo cuidado de forma segura e de boa qualidade.

Para Souza (2013), essa mudança de processos se torna necessária e as organizações devem concentrar os seus esforços na busca por mais informações de mercado, de modo que, com os seus processos sistematizados, possam almejar a sobrevivência, além de garantir a qualidade dos processos assistenciais em saúde. Nesse sentido, para a melhora dos processos, é necessário o envolvimento da gestão por meio do planejamento estratégico, com metas objetivas e possíveis, visando ao sucesso das atividades e à saúde financeira da organização.

Em contrapartida, Lorenzetti, Gelbcke e Vandresen (2016) discutem essa modificação de processo como desafiadora, uma vez que, das instituições contemporâneas, o hospital é percebido como um dos mais resistentes a mudanças. Isso ocorre devido ao baixo grau de interação entre as profissões e os departamentos, com fragmentação da prática clínica, grande subordinação dos usuários aos serviços e pouco governo dos gestores para atuar sobre as corporações. No que diz respeito à gestão de OPMEs, Lima (2015) afirma que manter os processos eficientes, com medição e monitoramento, torna-se imprescindível para evitar despesas desnecessárias e buscar maximização da receita, pois além do alto custo para a instituição de saúde, possuem armazenamento em estoque próprio ou de terceiros e possuem controle rigoroso por parte de operadoras de planos de saúde e Agência Nacional de Vigilância Sanitária (ANVISA).

Em uma pesquisa realizada por Nardino, Dalcul e Gil (2011), fez-se um estudo de caso do gerenciamento do setor de suprimentos de um hospital privado, no que se refere à distribuição de OPMEs. Constatou-se que esses itens são uns dos componentes de maior peso na fatura hospitalar. Contudo, o processo envolvendo o gerenciamento, apesar de bastante complexo, mostrou ter uma boa relação custo-benefício, sendo possível obter uma lucratividade de 14,3\% no período estudado. Embora apresente alguns gargalos, dentre os quais se destaca o tempo despendido durante o fluxo de OPME, o hospital consegue ter um controle do fluxo e do estoque que promove a viabilidade e os benefícios da utilização de processos gerenciais específicos na administração de OPME.

Porém, Camargo (2017) mostra outra realidade ao analisar, em hospital público, os custos dos dispositivos de OPME exclusivamente relacionados ao ato cirúrgico não incluídos na tabela SIGTAP. Nesse estudo específico, também foram identificados 11 dispositivos médicos não incluídos na tabela SUS, que corresponde a 146 (3\%) do total de internações com uso de OPME. Além disso, foi identificado que os custos das internações e procedimentos que envolveram os dispositivos de OPME na instituição hospitalar foram superiores aos valores pagos pelo SUS. Para o autor, além de a tabela SIGTAP não contemplar todos os dispositivos de OPME utilizados nas instituições atualmente, o pagamento realizado pelo SUS para os procedimentos executados não cobre todas 
as despesas necessárias, gerando um déficit financeiro para a instituição.

Buscando melhorar a qualidade da assistência oferecida e favorecer a sobrevivência financeira das instituições, algumas estratégias de gestão já vêm sendo implementadas. Um exemplo disso é o estudo realizado por Moraes, Rabin e Viégas (2018) que avaliou as falhas potenciais no processo de OPME, utilizando a ferramenta metodológica FMEA (Análise dos Modos de Falha e seus Efeitos) em um hospital de alta complexidade. A partir da revisão do fluxograma de OPME da instituição, os pesquisadores analisaram as etapas relacionadas à gestão de OPME, em busca de falhas que determinassem risco assistencial ao paciente. Todas as etapas analisadas apresentaram pelo menos um modo de falha potencial, totalizando 16 falhas e nenhuma foi classificada como alto risco. A partir desse estudo, foram propostas ações de melhoria para a gestão, como: padronização dos cadastros de materiais no sistema de informação; criação de um formulário específico para a solicitação de material; contratação de pessoal técnico especializado e criação de um programa de educação permanente.

Lima (2015) avaliou a adoção do monitoramento em tempo real (BAM) com o gerenciamento de regras de negócios (BPM) via sistema em processos de gestão administrativa de OPME, mais especificamente no contexto da sua comercialização. Percebeu-se que a estratégia utilizada pode qualificar fortemente a gestão, tanto na perspectiva tática quanto na estratégica e que a tecnologia da informação tem participação forte nessa empreitada da efetivação do BPM nas instituições. Como benefício da utilização do BPM, destacaram-se a qualificação das informações geradas pelo sistema informatizado de gestão e a identificação do cerne do problema da contabilização de OPME.

Na perspectiva da utilização da tecnologia da informação para a gestão em saúde, Pinochet, Lopes e Silva (2014) discutem sobre as novas tendências emergentes que estão trazendo benefícios diretos e indiretos nessa área. Para os autores, com relação à gestão no controle de estoque de materiais e medicamentos, a utilização de processos informatizados possibilita a redução de custos, por meio da redução de retrabalho, redução ou eliminação de roubos de materiais/medicamentos, fonte para geração de indicadores do hospital, apoiando o processo decisório e estratégico da gestão administrativa.

Para Wang et al. (2018), a tecnologia da informação, com base nos sistemas de informação em saúde, tem ganhado bastante evidência nos últimos anos, particularmente por oportunizar a redução de erros clínicos, como erros de medicação, erros de diagnóstico e para apoiar os profissionais de saúde oferecendo informações atualizadas. Eles prometem melhorar fluxo de trabalho e a eficiência dos cuidados, aumentando, assim, a qualidade geral de cuidados de saúde.

No estudo realizado por Assis e Miraldo (2015), foi apresentada a implantação de um sistema de gestão de processos, denominado "workflow", em uma operadora de saúde para controle de liberação de procedimentos de internações eletivas com a utilização de OPME. Como resultado, foi verificado que o sistema contribuiu para a eficiência do controle das solicitações de internações eletivas e no controle de materiais do tipo OPME, tendo apresentado como resultados o aumento de produtividade na ordem de 18\%. Isso possibilitou também a criação de indicadores que permitiram aos gestores monitorar a operação em tempo real, a emissão de relatórios históricos e a rápida recuperação dos documentos do processo.

Em outro estudo, Marquez-Peiro et al. (2016) descreveram a introdução do Sistema deVigilância de Produtos Sanitários, o SIVIPS, em um serviço de farmácia para o gerenciamento de dispositivos médicos no que diz respeito ao controle de uso e de incidentes. Um ano após sua implementação, foram 
registrados 564 implantes com uma descrição por tipo, 31 alertas e 6 incidentes. Foi verificado que a introdução da ferramenta de software, SIVIPS, permitiu uma melhora na rastreabilidade de próteses e implantes, e facilitou a coleta de dados sobre tipos de próteses de mais consumo e principais fornecedores, melhorou o registro e o monitoramento de incidentes relacionados com produtos sanitários, sendo uma informação básica para futuras decisões de aquisição de certas marcas de produtos ou fornecedores de produtos de saúde. Em um estudo semelhante, Mei e Lu (2016) desenvolveram um sistema para gerenciar a rastreabilidade de dispositivos médicos. Nesse estudo, os autores avaliaram o sistema como bom no gerenciamento de rastreabilidade e na unificação de processos.

Em um estudo publicado em 2016, Lorenzetti, Gelbcke e Vandresen desenvolveram um software de gestão para unidades de internação que abrangia diversos modos para gestão, entre eles, a de materiais. Nesse estudo, não há detalhes com relação a esse módulo, mas como resultado, os autores evidenciam, após análise de um ano de aplicação, benefícios na gestão de materiais, sendo a tecnologia avaliada positivamente pela equipe de enfermagem e pela comissão externa de avaliação.

Com relação aos desafios na adoção da tecnologia da informação, Wang et al. (2018) afirmam que a área da saúde tem sido particularmente lenta e atrasada em comparação com outras áreas. Isso é devido à complexidade em questões como interoperabilidade, racionalidade tecnológica, aceitabilidade, racionalidade gerencial, segurança de dados e qualidade dos dados. Essas limitações podem ser atribuídas a fatores técnicos, humanos e organizacionais. Nesse processo, a implementação de sistemas de informação em organizações de saúde representa uma série de desafios. Entretanto, para que haja um gerenciamento efetivo, é necessário ter o suporte de um sistema de informações que forneça tempestivamente os dados necessários ao processo decisório da logística e, consequentemente, possam ser avaliadas as alternativas para mais bem atender o cliente (MOURA; BEUREN, 2003).

Nessa mesma perspectiva, Burmester (2017) afirma que o sucesso dessas organizações, seja público, seja privado, cada vez mais, depende da capacidade de captar e processar as mais variadas informações, nos ambientes internos e externos, promovendo a organização e integração das partes interessadas, de modo sistematizado e oportuno, tornando-as em instrumentos importantes no processo decisório. Diante do exposto, apesar dos avanços ainda necessários nessa área, percebe-se que, para o gerenciamento eficiente de todo o processo envolvido na utilização dos OPMEs pelas instituições de saúde, é fundamental um suporte informacional ao processo decisório da logística, o que ocorre por meio dos sistemas de informações. $\bigcirc$ bom gerenciamento das OPMEs supera os benefícios em nível de gestão hospitalar quando atinge o objetivo da realização do procedimento com a satisfação do cliente atendido (FERREIRA JUNIOR, 2013).

No entanto, quando se trata de qualidade, a gestão de OPME deve estar em acordo com as legislações vigentes, devendo estas ser adotadas e seguidas de acordo com as estratégias gerenciais das instituições de saúde (BRASIL, 2016). Para Ferreira Junior (2013), isso proporciona aos usuários uma assistência de qualidade e sustentável, preservando a boa relação com os profissionais de saúde, pautada pela ética e transparência.

\section{CONCLUSÕES}

Baseados nos estudos supramencionados, podemos concluir que:

- As instituições hospitalares apresentam grande fragilidade gerencial, refletindo 
em combinação de altos custos e baixa qualidade na assistência à saúde, o que tem gerado a necessidade de iniciativas na busca por transformação no modelo de gestão e organização dos serviços de saúde. Para isso, o grande desafio é superar a impermeabilidade que as instituições hospitalares possuem no que diz respeito a mudanças.

- Com relação à gestão de OPME, existem portarias, manuais e instrumentos que orientam a gestão. Contudo, ainda é possível verificar que são diversas as dificuldades que interferem na prática. Dentre elas, podemos destacar: falta de informação de qualidade e conflitos de interesse entre os atores, ausência de controle de estoque, não padronização dos processos, desperdícios e má utilização desses materiais, baixa qualificação dos profissionais e o ineficiente planejamento logístico nas organizações de saúde.

- Especificamente em instituições públicas, as barreiras de legislação que restringem a agilidade necessária, a alta rotatividade dos gestores, a ausência de diversas OPMEs na tabela SIGTAP e o pagamento insuficiente para cobrir as despesas para os procedimentos executados dificultam ainda mais 0 gerenciamento de OPMEs e geram um déficit financeiro para a instituição.

- A melhora da qualidade da assistência oferecida e a sobrevivência financeira das instituições hospitalares são preocupações presentes entre os profissionais de saúde. A padronização de processos, o controle do fluxo e do estoque e a capacidade de captar e processar as informações pertinentes ao processo de trabalho são requisitos fundamentais no gerenciamento de OPME.

- Como tendência, observou-se claramente que a utilização da tecnologia da informação, por meio dos sistemas de gestão em saúde, é o caminho a ser adotado. Essa afirmação é fundamentada no fato de que a pesquisa realizada mostrou que os hospitais com os modelos de gestão conjugados com eficientes sistemas de TI tiveram os seguintes benefícios: melhoria do gerenciamento de OPMEs, melhorias relacionadas ao fluxo de comunicação, padronização de processos, produção de indicadores para a gestão, rastreabilidade de dispositivos e redução dos custos. Além disso, foi observado um maior controle no fluxo de trabalho, minimizando os riscos de roubos e fraudes no faturamento. 


\section{REFERÊNCIAS}

AGUIAR, F. C.; MENDES, V. L. P. S. Comunicação Organizacional e Tecnologias da Informação e Comunicação (TICs) na Gestão Hospitalar. Perspectivas em Ciência da Informação, v. 21, n. 4, p. 138-155, 2016.

ALENCAR, A. C. F. Aquisição e utilização das Órteses, Próteses e Materiais Especiais - OPME e os facilitadores do superfaturamento no sistema de saúde. 2016. Trabalho de Conclusão de Curso (Monografia) - Universidade de Brasília, Brasília, 2016.

ALSHAGATHRH, F. et al. Building a cloud-based data sharing model for the Saudi national registry for implantable medical devices:

Results of a readiness assessment. Int J Med Inform, v. 118, p. 113119, 2018.

ALVES, J. L. de; GUERRA NETO, C. L. B.; HÉKIS, H. R. A importância de indicadores de qualidade em serviços de hemoterapia. In: GUERRA NETO, C. L. B. et al. (org.). Gestão e Inovação em Saúde: O que estamos fazendo na EBSERH. 1. ed. Natal: SEDIS-UFRN, 2018. p. 141-170.

ASSIS, S. F. M.; MIRALDO, C. O. Implantação de um sistema informatizado para gerenciamento da liberação de cirurgias com controle de órteses, próteses e materiais especiais em uma operadora de saúde. In: SEMEAD - SEMINÁRIOS EM ADMINISTRAÇÃO. 18., 2015. São Paulo. Anais [...]. São Paulo: FEAUSP, 2015.

AZEVEDO, C. S. et al. Racionalização e Construção de Sentido na Gestão do Cuidado: uma experiência de mudança em um hospital do SUS. Ciênc. saúde colet., v. 22, n. 6, p. 1991-2002, 2017.

BRASIL. Ministério da Saúde. Agência Nacional de Vigilância Sanitária. Agência Nacional de Saúde Suplementar. Relatório final do Grupo de Trabalho Externo de Órteses, Próteses e Materiais Especiais (GTE OPME). Rio de Janeiro: Ministério da Saúde, 2016. Disponível em: http://www.ans.gov.br/images/stories/Particitacao_ da_sociedade/2016_gt_opme/gt-opme-relatoriointegral.pdf. Acesso em: 5 fev. 2019.

BRASIL. Ministério da Saúde. Grupo de Trabalho Interinstitucional sobre órteses, próteses e materiais especiais (GTI-OPME). Relatório Final. Brasília: Ministério da Saúde, 2015. Disponível em: http:// portalarquivos.saude.gov.br/images/pdf/2015/julho/07/RelatorioFinal-versao-final-6-7-2015.pdf. Acesso em: 5 ago. 2019. 
BRASIL. Ministério da Saúde. Manual de Boas Práticas de Gestão das Órteses, Próteses e Materiais Especiais (OPME). Brasília: Ministério da Saúde, 2016. Disponível em: http://bvsms.saude.gov. br/bvs/publicacoes/manual_praticas_gestao_proteses_materiais_ especiais.pdf. Acesso em: 15 out. 2019.

BRASIL. Ministério da Saúde. Portaria n. 1.302, de $1^{\circ}$ de agosto de 2017. Redefine os critérios para aquisição, recebimento, utilização, monitoramento, controle e gerenciamento de OPME pelos hospitais e institutos federais subordinados à Secretaria de Atenção à Saúde do Ministério da Saúde (SAS/MS). Brasília: Ministério da Saúde, 2017. Disponível em: http://lex.com.br/ legis_27480779_PORTARIA_N_1302_DE_1_DE_AGOSTO_DE_2017. aspx. Acesso em: 25 ago. 2019.

BURMESTER, H. Gestão da qualidade hospitalar. São Paulo: Saraiva, 2017.

\section{CAMARGO, T. A. C. Custos de órteses, próteses e materiais} especiais não contemplados no Sistema Único de Saúde (SUS) em hospital de ensino brasileiro. 2017. Dissertação (Mestrado) Faculdade de Medicina, Universidade Estadual Paulista, Botucatu, 2017.

CAÑAMARES-ORBIS, l. et al. Implantación de un sistema de trazabilidad en el área de farmacia oncológica en un hospital de tercer nivel. Revista de Calidad Asistencial, v. 1, n. 37, p. 56-76, 2014.

CRUZ, T. Sistemas de informações gerenciais: tecnologias da informação e a empesa do século XXI. 3. ed. São Paulo: Atlas, 2011.

DEUS, A. D.; MELO, E. M. Avaliação de uma experiência de gestão hospitalar participativa no âmbito do SUS: produção de saúde, sujeitos e coletivos. Saúde debate, v. 39, n. 106, p. 601-615, 2015.

EDELSTEIN, J. E.; BRUCKNER, J. Órteses: abordagem clínica. Rio de Janeiro: Guanabara Koogan, 2006.

FARIAS, D. C., ARAUJO, F. O.de. Gestão hospitalar no Brasil: revisão da literatura visando ao aprimoramento das práticas administrativas em hospitais. Ciênc. saúde coletiva, v. 22, n. 6, p. 1895-1904, 2017.

FERREIRA JUNIOR, W. C. OPME - Órteses, Próteses e Materiais Especiais: uma discussão sobre usos e abusos. Debates GVsaúde, v. 15, p. 16- 29, 2013. 
FONSECA JÚNIOR, A. L. T. Kryptotag: Etiqueta baseada no protocolo Blockchain Ethereum para Órteses, Próteses e Materiais Especiais (OPME). 2018. Dissertação (Mestrado) - Escola Paulista de Medicina, Universidade Federal de São Paulo, São Paulo, 2018.

GARCIA, S. D. et al. Gestão de material médico-hospitalar e o processo de trabalho em um hospital público. Revista Brasileira de Enfermagem, v. 65, n. 2, p. 339-346, 2012.

GIJO, E. V.; ANTONY, J. Reducing Patient Waiting Time in Outpatient Department Using Lean Six Sigma Methodology. Qual.

Reliab. Engng. Int, v. 30, p. 1481-1491, 2014.

ITO, V. Custo com prótese vai a R $\$ 20$ bilhões ao ano e pressiona operador de saúde. Diário Comércio indústria e serviços. 2015. Disponível em: http://www.capesesp.com.br/ noticias/- /asset_publisher/7jll/content/custo-com-protesevai-a-r-20-bilhoes-ao-anoe-pressiona-operador-desaude/ maximized;jsessionid=F09591F8388AC314CCC8910DDA66CF12 . 2017. Acesso em: 18 nov. 2017.

KUSHNIRUK, A. W.; PATEL, V. L. Cognitive and usability engineering methods for the evaluation of clinical information systems. Journal of Biomedical Informatics, v. 37, n. 1, p. 56-76, 2004.

LAUDON, K. C.; LAUDON, J. P. Sistemas de informação gerenciais. São Paulo: Pearson Prentice Hall, 2007.

LIMA, L. Gestão da comercialização de OPME: um estudo de caso de monitoramento de processo de negócio em tempo real. 2015. Trabalho de Conclusão de Curso (Especialização) - Unidade Acadêmica de Educação Continuada, Universidade do Rio dos Sinos, Porto Alegre, 2015.

LORENZETTI, J.; GELBCKE, F. L.; VANDRESEN, L. Tecnologia para gestão de unidades de internação hospitalares. Texto Contexto Enferm, v. 25, n. 2, 2016. Disponível em: http://www.scielo.br/pdf/ tce/v25n2/pt_0104-0707-tce-25-02-1770015.pdf. Acesso em: 21 jan. 2019.

MARÇULA, M.; BENINI FILHO, P. A. Informática: conceitos e aplicações. 4. ed. São Paulo: Érica, 2013.

MARQUEZ-PEIRO, J. F. et al. Diseño de un aplicativo para el registro de implantes, gestión de alertas e incidentes relacionados con productos sanitarios como herramienta para el responsable de vigilancia de productos sanitarios. Farm Hosp, v. 40, n. 2, p. 118-123, 2016. 
MEI, X.; LU, Y. Development of Implantable Medical Device

Traceability Management Information System. Zhongguo Yi Liao Qi

Xie Za Zhi, v. 40, n. 5, p 359- 362, 2016.

MORAES, C. S. Análise do processo de órteses, próteses e materiais especiais a partir do método de análise dos modos de falhas e efeitos. 2014. Dissertação (Mestrado) - Escola Superior de Saúde, Universidade do Vale do Rio dos Sinos, Porto Alegre, 2014.

MORAES, C. S.; RABIN, E. G.; VIÉGAS, K. Assessment of the care process with orthotics, prosthetics and special materials. Revista Brasileira de Enfermagem, v. 71, n. 3, p. 1099-105, 2018.

MOURA, V. M.; BEUREN, I. M. O Suporte Informacional da Controladoria para o Processo Decisório da Distribuição Física de Produto. Revista Contabilidade \& Finanças. USP. São Paulo, n. 31, p. 45-65, jan./abr. 2003.

NARDINO, S.; DALCUL, A. L.; GIL, P. Controle de estoque de OPME. Science in Health, São Paulo, v. 2, n. 2, p. 113-119, 2011. Disponível em: http://arquivos.cruzeirodosuleducacional.edu.br/principal/new/ revista_scienceinhealth/05_maio_ago_2011/science_113_119.pdf. Acesso em: 22 jan. 2019.

NEUFELD, P. M. Uma breve história dos hospitais. Revista Brasileira de Análises Clínicas, v. 5, n. 4, p. 7-13, 2013.

NORTON, K. M. A Brief History of Prosthetics. In Motion, v.17, n.7, 2007. Disponível em: https://3w568y1 pmc7umeynn2o6c1mywpengine.netdna-ssl.com/wp-content/uploads/2015/03/ history_prosthetics.pdf. Acesso em: 05 ago. 2019.

OECD/EUROSTAT. Oslo Manual 2018: Guidelines for Collecting, Reporting and Using Data on Innovation, 4th Edition, The Measurement of Scientific, Technological and Innovation Activities, OECD Publishing, Paris/Eurostat, Luxembourg, 2018. Disponível em: https://www.oecd.org/sti/inno/oslo-manual-2018-info.pdf. Acesso em: 22 abr. 2019.

\section{PEREIRA, L. M. V. Gestão da farmácia hospitalar e a percepção} dos sujeitos. 2015. Tese (Doutorado) - Faculdade de Medicina, Universidade de São Paulo, Ribeirão Preto, 2015.

PINOCHET, L. H. C.; LOPES, A. S.; SILVA, J. S. Inovações e Tendências Aplicadas nas Tecnologias de Informação e Comunicação na Gestão da Saúde. Revista de Gestão em Sistemas de Saúde - RGSS, v. 3, n. 2, p. 11-29, 2014. 
RAIMUNDO, E. A.; DIAS, C. N.; GUERRA, N. Logística de medicamentos e materiais em um hospital público do distrito federal. Revista de Administração Hospitalar e Inovação em saúde, v. 12, n. 2, p. 61-69, 2015.

\section{REIS, M. P. M. Gestão estratégica da cadeia de abastecimento}

hospitalar: Análise do fluxo logístico de materiais médicohospitalares em uma unidade assistencial da FHEMIG. 2017.

Trabalho de Conclusão de Curso (Monografia) - Escola de Governo Professor Paulo Neves de Carvalho, Fundação João Pinheiro, Belo Horizonte, 2017.

\section{SCHROEDER, L.; SCHIOCHET, R. A. Criação da gestão de OPME} com foco na armazenagem: um estudo de caso no Hospital Municipal São José de Joinville - SC. 2018. Trabalho de Conclusão de Curso (Monografia) - Instituto Federal de Santa Catarina, Joinville, 2018.

SEIXAS, M. A. S.; MELO, H. T. Desafios do administrador hospitalar. Revista Gestão e Planejamento, Salvador, v. 5, n. 9, p. 16-20, 2004.

SILVA, J. N. A.; LIMA, J. C. S. Gestão de materiais: almoxarifado hospitalar. Cad. Unisuam Pesqui. Ext., v. 5, n. 4, p. 35-45, 2015. Disponivel em: http://apl.unisuam.edu.br/revistas/index.php/ cadernosunisuam/article/view/831/664 Acesso em: 16 jul. 2019.

SILVA, L. F. et al. Vulnerabilidade e riscos de ruptura no abastecimento de materiais e medicamentos na cadeia de suprimento em um Hospital Público. GEPROS. Gestão da Produção, Operações e Sistemas, ano 13, n. 2, p. 21-43, 2018.

SILVA, T. O. da et al. Gestão hospitalar e gerenciamento em enfermagem à luz da filosofia Lean Healthcare. Cogitare enferm. 2019. Disponível em: http://dx.doi.org/10.5380/ce.v24i0.60003. Acesso em: 24 jun. 2019.

SOUZA, M. A. Análise Comparativa dos Indicadores de Qualidade Implantados nos Hemocentros de Referência do Brasil. 2013. 108 f. Trabalho de Conclusão de Curso (Monografia) - Faculdade de INESP, Florianópolis, 2013.

SOUZA, V. P.; CARVALHO, R. R. Gestão do conhecimento no âmbito da administração hospitalar: proposta de modelo conceitual integrativo para gestão do corpo clínico. Revista de Gestão em Sistemas de Saúde - RGSS, v. 4, n. 2, p. 97-112, 2015. Disponível em: https://dialnet.unirioja.es/servlet/articulo?codigo $=5294588$. Acesso em: 22 maio 2019. 
VIEIRA, L.V. Inovação no setor público: indutores, capacidades, tipos e resultados de inovação. 2016. Dissertação (Mestrado) Programa de Pós-Graduação em Administração, Universidade de Brasília, Brasília, 2016.

VILELA PINTO, L. F.; NOSSA, V.; TEIXEIRA, A. Inovação: estratégia de competitividade e sustentabilidade na gestão hospitalar.

CONTEXTUS - Revista Contemporânea de Economia e Gestão, v. 13, n. 3, p. 216-243, 2015.

WANG, Y. C. et al. Increased Risk of Dementia in Patients with Antidepressants: A Meta-Analysis of Observational Studies.

Behavioural Neurology, v. 1, p. 1-8, 2018. 